\title{
A wide-range micromachined threshold accelerometer array and interface circuit
}

\author{
Arjun Selvakumar ${ }^{1}$, Navid Yazdi $^{2}$ and Khalil Najafi ${ }^{3}$ \\ ${ }^{1}$ Input/Output, Inc., Stafford, TX, USA \\ ${ }^{2}$ Arizona State University, Tempe, AZ, USA \\ ${ }^{3}$ Center for Integrated Microsystems, University of Michigan, Ann Arbor, MI 48109-2122, \\ USA
}

Received 3 July 2000, accepted for publication 20 July 2000

\begin{abstract}
This paper presents a complete threshold acceleration detection microsystem comprising an array of threshold accelerometers and a low power interface circuit. The sensors were designed and fabricated using the bulk-silicon dissolved-wafer process. The process offers a wide latitude in sensor threshold levels, as demonstrated in the fabrication of devices with levels of $1.5-1000 \mathrm{~g}$, bandwidths of $45 \mathrm{~Hz}$ to $40 \mathrm{kHz}$, with mass sizes ranging from $0.015 \mu \mathrm{g}$ to $0.7 \mu \mathrm{g}$, and low-resistance gold-gold contacts for the switch. The interface circuit dissipates less than $300 \mu \mathrm{W}$, measures $2.2 \mathrm{~mm} \times 2.2 \mathrm{~mm}$; it was fabricated in-house using a standard $3 \mu \mathrm{m}$, p-well CMOS (complementary metal oxide semiconductor) process, and is connected to the sensor chip in a multi-chip module. The key aspects of the microsystem are the implementation of sensor redundancy and supporting circuit logic to improve detection accuracy and fault tolerance, which are crucial factors in many applications. In addition, the microsystem supports communication with a standard microcontroller bus in a smart sensor network.
\end{abstract}

\section{Introduction}

Accelerometers are required in many applications in which force, acceleration, vibration and displacement are to be sensed [1]. Some of these applications, in particular those concerning munition fusing, seat-belt pre-tensioners, microrelays and airbag deployment, require sensors that will switch electrical states using the detection of a preset threshold acceleration level. Due to the inherent nature of these applications, functional specifications of the microsystem require high accuracy, repeatability, reliability, miniaturization and ease of interface with the host microprocessor. From a manufacturing standpoint, since most of these applications predominantly fall into the commodities sector, low cost and batch fabrication are also essential. With this preface, microsystems employing silicon micromachined accelerometers and supporting circuitry are a viable choice.

While there has been considerable effort put into microaccelerometers measuring continuously varying acceleration, much less has been published on applications with MEMS (micro electro mechanical systems) measuring shock or threshold acceleration levels [2-5]. Even less has been done on such microsystems addressing fault tolerance and measurement integrity, especially where human lives are at stake. Increasingly, in many applications, such as portable micro-instrumentation systems, a limited power supply necessitates low power consumption. Thus, there is a need for a low cost, low power system that addresses these issues and can be designed over a wide range, for use in various applications.

This paper presents a threshold-acceleration-sensing system that employs silicon micromachined accelerometers, interfaced with a CMOS (complementary metal oxide semiconductor) detection circuitry [6]. The accelerometers function as switches that are triggered by accelerations greater than the threshold level of the selected sensor. The interface circuit captures the sensor output transition, and provides a digital output of the event. Furthermore, the system employs sensors replicated thrice at each threshold level to provide adequate redundancy. The output of the sensor system is weighted on the majority status of these redundant sensors. Thereby, fault tolerance and an improved overall accuracy is obtained.

The sensor chip is fabricated using a modified dissolvedwafer process allowing batch fabrication using a silicon and glass wafer bonded pair. The process is flexible offering the modification of many design parameters, and enabling the design of a wide range of threshold accelerometers. 
The simplicity and batch fabrication of the process technology enables the low cost of the sensor die. The circuit chip is fabricated in-house using a single polysilicon, single metal, $3 \mu \mathrm{m} \mathrm{p}$-well CMOS process. The following sections will describe the sensor operation, circuit description, the sensor fabrication, the measurement results and the conclusions.

\section{Sensor design and operation}

The threshold accelerometer consists of an inertial mass, which is suspended by a compliant spring, and separated from the support frame by an air-gap. A shock or an acceleration greater than the threshold of the device causes the mass to deflect, traversing the air gap, and making contact with the frame. A metal strip on the bottom surface of the inertial mass shorts metal contacts on the frame and closes a sensor circuit, thus making the sensor act as an event trigger or switch. The threshold acceleration level is essentially a function of the mass of the inertial element, the spring constant of the suspension, and the dimension of the air-gap separating the contacts on the mass and the frame. By altering one or more of these design parameters, different thresholds can be achieved to suit a given application.

The accelerometer described here is constructed using a modified bulk-silicon dissolved-wafer fabrication process [7] with a $15 \mu \mathrm{m}$ thick $\mathrm{p}^{++}$inertial mass suspended by a $7500 \AA$ silicon oxide suspension beam, anchored to a glass substrate. Different thresholds are achieved by varying the layout area of the inertial mass and the spring. The device employs gold switch-contacts on both the $\mathrm{p}^{++}$mass and the glass substrate, as shown in detail in figure 1 separated by $0.25 \mu \mathrm{m}$. It is desirable to produce a suspension that has a low spring-constant such that the size of the inertial mass for a given threshold can be reduced, hence reducing die size and achieving device scalability. Moreover, a softer spring facilitates the measurement of smaller values of acceleration (in the range of a few 'g's), which would otherwise prove to be difficult obtaining the necessary deflection to close the switch. These spring design objectives are achieved by employing an LPCVD (low-pressure chemical vapour deposition) silicon-oxide spring beam. LPCVD processes provide ease in deposition control of small layer thickness (tens of nm level control). Moreover, the relatively low Young's modulus of silicon oxide $\left(0.7 \times 10^{11} \mathrm{~N} \mathrm{~m}^{-2}\right)$ leads to a lower spring-constant than most other MEMS material choices. A cantilevered beam design was chosen for the spring-constant per unit die area being relatively lower than other suspensions designs and the beam being able to release residual stress through axial elongation or compression at the free-end.

Under the influence of an acceleration above the threshold value, the device, being cantilevered, deflects in such a manner that the leading edge of the mass comes into contact with the frame. The acceleration versus deflection expression for a similarly cantilevered structure is given in [8] and is modified in equation (1) to express threshold acceleration $a_{t}$ as a function of material and design parameters:

$$
a_{t}=\frac{E_{O x} w_{b} t_{b}^{3} \delta_{g}}{\rho_{S i} w_{m} t_{m} l_{m} l_{b}^{3}}\left(\frac{1}{4+9 \alpha+6 \alpha^{2}}\right)
$$

where $l_{m}, w_{m}, t_{m}, l_{b}, w_{b}, t_{b}$ are the length, width and thickness of the mass and beam respectively, $E_{O x}$ is the Young's modulus of the oxide beam, $\rho_{S i}$ is the density of silicon, $\delta_{g}$ is the air-gap dimension between the switch contacts, and $\alpha$ is the ratio of $l_{m}$ to $l_{b}$.

It can be observed that, in the cantilevered design, the length of the mass is an additional design parameter for setting the threshold level. Note that the threshold acceleration is inversely proportional to the third power of the beam length and, as such, it is a strong design parameter. Using this design, sensors with a wide range of thresholds can be fabricated with well-controlled sensor dimensions.

The operational bandwidth of the sensor was calculated assuming a squeeze-film damping mechanism with the sensor operated at atmospheric pressure. The resulting damping coefficients $b$ were calculated using the expression for a simple parallel-plate structure [9]. Although in reality the inertial mass rotates, the result based on assuming translational motion provides an upper limit for the damping coefficient and hence a conservative estimate for the operational bandwidth.

$$
b=f \mu \frac{l_{m}^{4}}{d^{3}}
$$

where $f$ is a form factor that takes into account the geometry, $\mu$ is the viscosity of air at room temperature and pressure and $d$ is the gap separating suspended sensor structure and the glass substrate. Squeeze-film damping between narrow gap $(1-2 \mu \mathrm{m})$ structures can typically produce over-damped conditions that reduce the bandwidth. In the design of these accelerometers, an additional recess etch step on the glass substrate is performed so that only the contacting points are in close proximity to each other as required by the design value of $\delta_{g}$. The remainder of the structure is separated by $d$, an adequately larger gap (7.5 $\mu \mathrm{m}$ was used in the sensor design) to suppress squeeze-film damping and increase the operational bandwidth without affecting the switching threshold of the accelerometer.

The $3 \mathrm{~dB}$ cut-off frequency $f_{c}$ was calculated from the theoretical transfer function of a lumped element, second-order system and given by (3)

$$
f_{c}=f_{n} \sqrt{\sqrt{4 \xi^{4}-4 \xi^{2}+2}-2 \xi^{2}+1}
$$

where the resonant frequency $f_{n}$ and the damping ratio $\xi_{n}$ are given by (4) and (5) respectively.

$$
\begin{aligned}
f_{n}= & \frac{1}{2 \pi}\left\{\frac{E_{O x} w_{b} t_{b}^{3}}{12 l_{m} w_{m} t_{m} \rho_{S i} l_{b}^{3}}\right. \\
& \left.\times\left[\frac{2+3 \alpha+0.25 \alpha^{2}}{0.67+2 \alpha+2.63 \alpha^{2}+1.75 \alpha^{3}+0.5 \alpha^{4}}\right]\right\}^{1 / 2} \\
\xi= & \frac{b}{4 \pi f_{n} \rho_{S i} l_{m} w_{m} t_{m}} .
\end{aligned}
$$

The dissolved-wafer process enables the sensors to employ a suitably sized, $15 \mu \mathrm{m}$ thick, $\mathrm{p}^{++}$silicon inertial mass. With this mass thickness and thin beams afforded by the LPCVD oxide beams, the sensors take up very little die area. Hence, a large number of threshold accelerometers can be designed on a single chip. Table 1 lists the range of thresholds that were chosen on the present design, with thresholds in ' $\mathrm{g}$ ' 


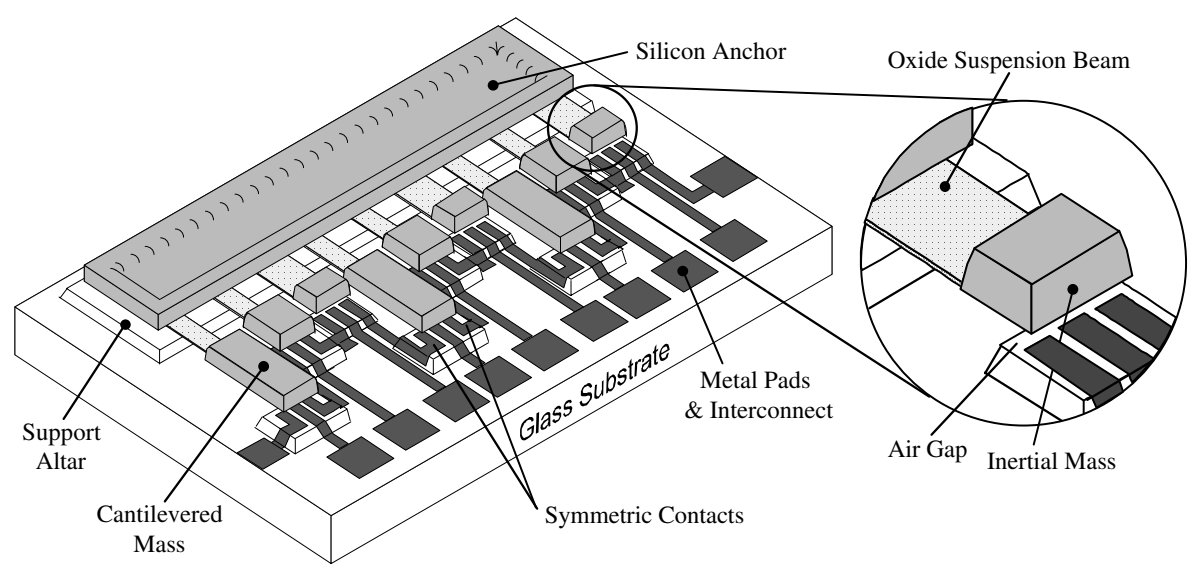

Figure 1. Illustration of threshold acceleration sensors employing redundancy (three identical sets, each set containing three different thresholds). The detail shows a single threshold switch with a cantilevered mass. An upward acceleration greater than the threshold of the device causes a gold strip (not shown) under the deflected mass to short the gold leads on the glass substrate.

Table 1. Threshold accelerometer designs. All beam thicknesses are $0.75 \mu \mathrm{m}$ and all mass thicknesses are $15 \mu \mathrm{m}$ except for the $1000 \mathrm{~g}$ device with a mass thickness of $2.5 \mu \mathrm{m}$. The separation between contacts is $0.25 \mu \mathrm{m}$.

\begin{tabular}{cclr}
\hline Threshold & $l_{b}, w_{b}(\mu \mathrm{m})$ & $l_{m}, w_{m}(\mu \mathrm{m})$ & $f_{c}(\mathrm{~Hz})$ \\
\hline $1.5 \mathrm{~g}$ & 100,50 & 175,114 & 45 \\
$2 \mathrm{~g}$ & 75,50 & 200,100 & 47 \\
$5 \mathrm{~g}$ & 75,50 & 100,100 & 670 \\
$10 \mathrm{~g}$ & 50,50 & 100,100 & 1120 \\
$50 \mathrm{~g}$ & 60,15 & 40,63 & 9918 \\
$100 \mathrm{~g}$ & 60,30 & 40,63 & 15085 \\
$1000 \mathrm{~g}^{\mathrm{a}}$ & 50,30 & 35,71 & 39793 \\
\hline
\end{tabular}

a $2.5 \mu \mathrm{m}$ thick mass.

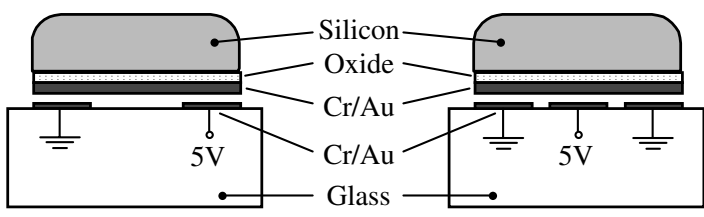

(a)

(b)

Figure 2. Front view of the threshold switch is shown: (a) asymmetric pull down with double contact configuration; and (b) balanced pull down with triple contact layout.

units $\left(1 \mathrm{~g}=9.8 \mathrm{~m} \mathrm{~s}^{-2}\right)$ and $f_{c}$, the cut-off frequency of the accelerometer. A wide range of thresholds (1.5-1000 g) was designed. Due to its high threshold value, the $1000 \mathrm{~g}$ threshold accelerometer design required a smaller inertial mass thickness than feasible with the $15 \mu \mathrm{m}$ thick $\mathrm{p}^{++}$structural layer. For this particular design, a shallow boron doping profile was employed to create a $2.5 \mu \mathrm{m}$ deep $\mathrm{p}^{++}$etch stop layer to limit the thickness of the inertial mass to $2.5 \mu \mathrm{m}$. This enabled a $83 \%$ reduction in the inertial mass design for a given area over the thicker deep boron diffusion etch stop depth.

With the design dimensions listed in table 1, an array of accelerometers with various threshold was designed and implemented on the sensor mask sets. Sensors at each given threshold were replicated thrice such that, with 'majority weighting' circuit logic support, the redundancy feature of the microsystem is implemented. The increase in die area is feasible using the dissolved-wafer process owing

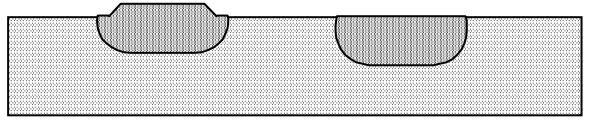

(a)

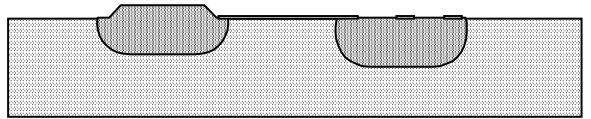

(b)

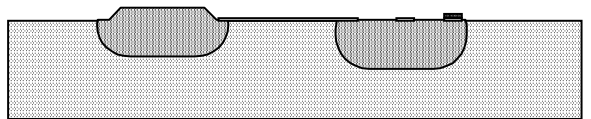

(c)

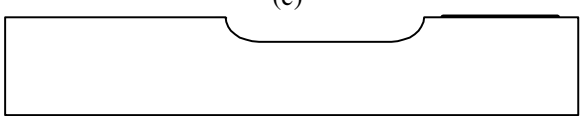

(d)

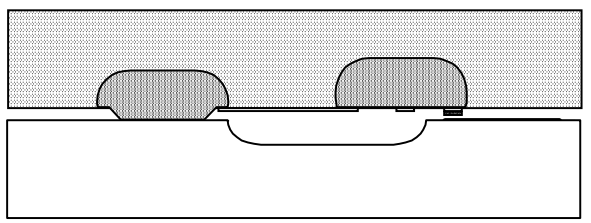

(e)

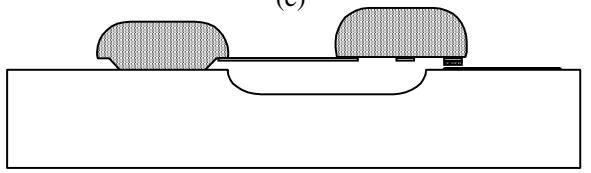

(f)

Figure 3. Process sequence for the fabrication of threshold accelerometers using a silicon and a glass wafer, with the modified dissolved wafer process. (For details see text.)

to the individual sensor area being quite small (refer to table 1). As noted before, this redundant usage improves the system reliability since if any of the sensors break or falter during operation, the system can still function with the redundant replicates. Figure 1 illustrates an array of threshold accelerometers of essentially three dissimilar designs with triple redundancy. 


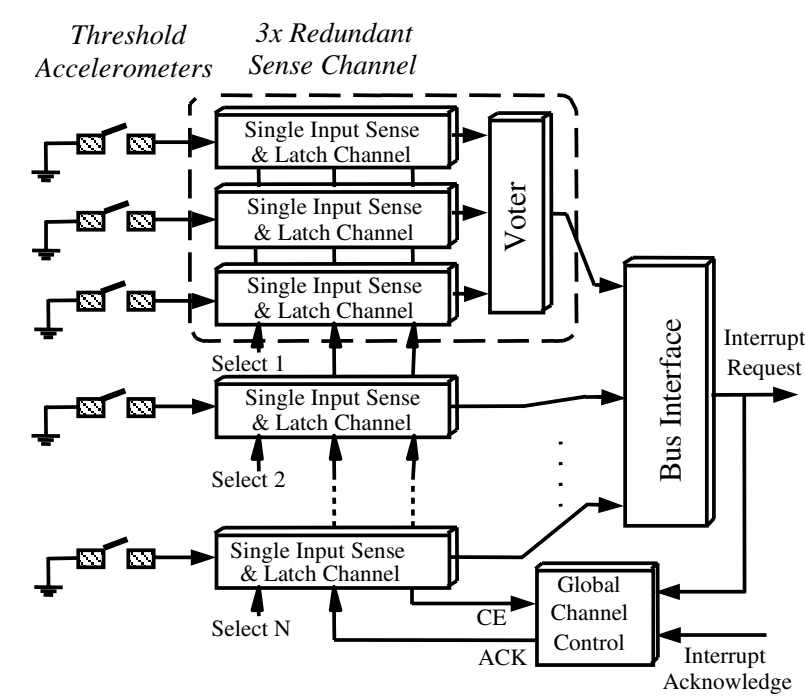

Figure 4. Functional circuit block diagram showing the input redundant channels and the logic voting of the majority function.

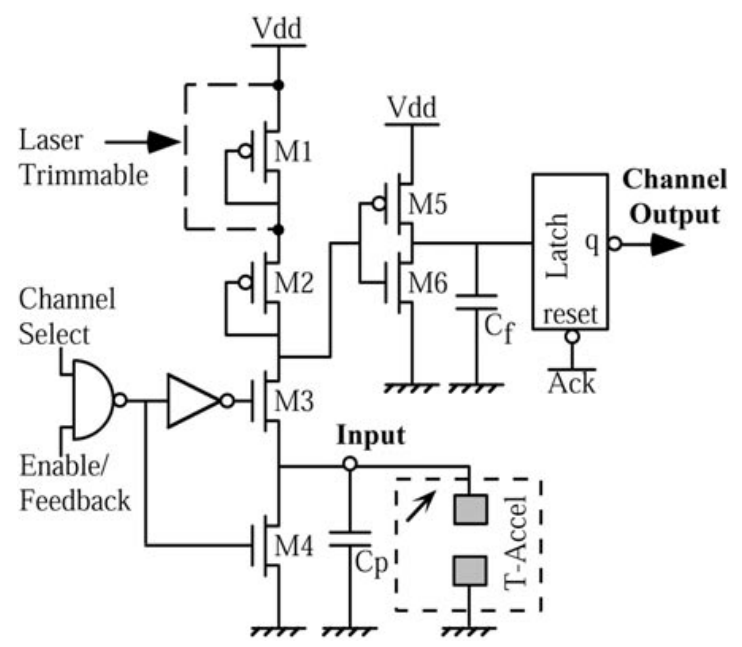

Figure 5. Schematic diagram of a sense channel.

The readout interface circuit applies a $5 \mathrm{~V}$ potential across the gold contacts on the glass substrate causing image charges to build up on the inertial mass contact points. The charge build-up produces an asymmetric electrostatic force of attraction that would pull the inertial mass unevenly towards the glass substrate. This would result in non-planar contacting between the switch contacts thus preventing a short across a 'two-point' contact configuration, as shown in figure 2(a). This is avoided by employing a electrostatically balanced configuration scheme with the two outer contacts at ground potential and the central contact at the $5 \mathrm{~V}$ potential, as illustrated in figure $2(b)$. This scheme allows a balanced pulldown when the inertial mass is at close proximity to the switch contacts. The outer ground contacts are routed and shared between each sensor in the array so as to minimize the number of bond pads by half.

The inclusion of metal pull-in electrodes on the glass substrate can be employed such that they will electrostatically pull-in and vary the air-gap. This enables one to tune the threshold acceleration levels after fabrication. This method can

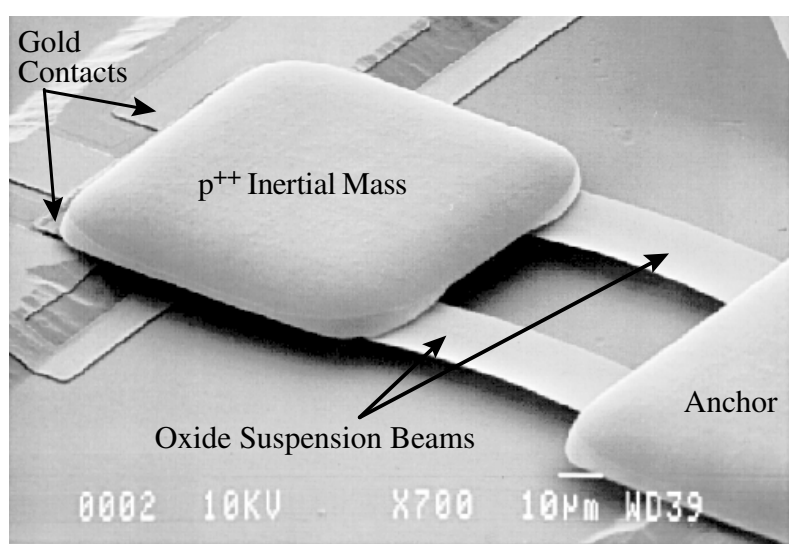

Figure 6. SEM micrograph of one of the threshold accelerometers $(10 \mathrm{~g})$, showing the thick $\mathrm{p}^{++}$inertial mass $(15 \mu \mathrm{m})$ suspended by a dual oxide beam $(50 \mu \mathrm{m} \times 25 \mu \mathrm{m})$ suspension. The gold contact under the inertial mass can be seen as well as the metal contacts on the glass substrate.

be extended to enable the threshold accelerometer to function as an electrostatically activated, micro-relay [2] offering a very high on/off resistance ratio. Moreover, employing an array of uniformly varying thresholds provides the option of having a digital accelerometer. The range and resolution of the accelerometer would then be determined by the thresholds of the individual accelerometers constituting the array.

\section{Sensor fabrication}

The sensor was fabricated using a modified bulk-silicon dissolved-wafer process. This process technology is simple, yet it offers flexibility over the control of the design parameters. It allows for the batch fabrication of sensors that are robust, improving process yield and decreasing device cost. Using this process, sensor elements are formed from heavily boron diffused regions, and an automatic end point, etch control is provided using EDP (ethylene diamine pyrocatechol) as an etchant. This process facilitates modification of the airgap, by simply altering the recess etch on the silicon wafer, using a well-controlled $\mathrm{KOH}$ (potassium hydroxide) etch step. The gap dimension can be suitably chosen such that with layout variations in the mass dimensions and the oxide beam dimensions, the desired threshold level can be met. A value of $0.25 \mu \mathrm{m}$ contact separation gap was used for the design calculations. This is achievable using a $1.5 \mu \mathrm{m} \mathrm{KOH}$ recess that is eventually reduced to the design gap value by the metal and LPCVD oxide structural layers. Although, the cantilevered design possesses a low spring-contact and the devices are more susceptible to breakage due to high shock, using the dissolved-wafer process, a second silicon wafer [10]—acting as a shock stop — can be easily provided to prevent sensor damage.

The process is a modified version of the dissolved-wafer process, as it employs a metal layer on the silicon wafer, as well as on the glass wafer. This is mandatory as it enables the fabrication of a switch with low contact resistance (gold) for both of the contacting surfaces of the switch, improving detection accuracy. An evaporated gold strip on the underside of the inertial element comes into contact with similarly deposited gold 


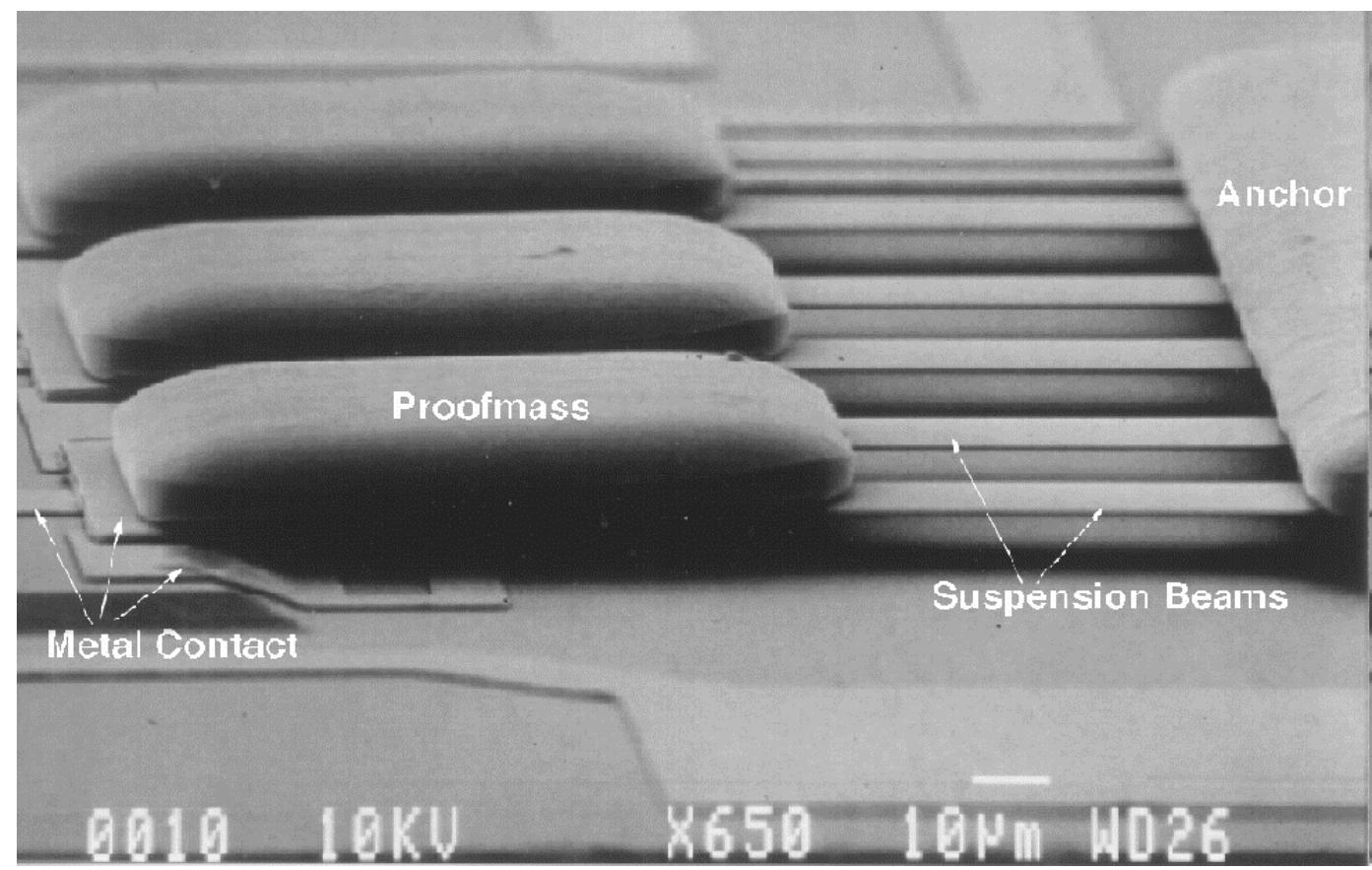

Figure 7. SEM micrograph of a triple array with threshold of $1.5 \mathrm{~g}$ with $100 \mu \mathrm{m}$ long $25 \mu \mathrm{m}$ wide and $0.75 \mu \mathrm{m}$ thick oxide beams and $175 \mu \mathrm{m}$ long, $114 \mu \mathrm{m}$ wide and $15 \mu \mathrm{m}$ thick inertial mass. The recess under the sensor is $7.5 \mu \mathrm{m}$ deep with a $1.5 \mu \mathrm{m}$ air-gap between the contacts.

leads on the glass substrate. Chromium is used to improve the adhesion of the gold to the silicon and glass. It is probable that the oxide beams may stick to the glass substrate upon post etch release of the structure. Therefore, a further modification of the process is to recess the glass, except in the anchoring and contacting region, so as to reduce this stiction-related failure.

The process consisted of a total of six masking stepsfour on the silicon wafer and two on the glass wafer-and is highlighted in figure 3(a)- $(f)$ starting with the silicon wafer. The first step was a $1.5 \mu \mathrm{m} \mathrm{KOH}$ anchor recess step that creates the air-gap for the switch. This can be modified to suit the range of interest of the application. Second, a $15 \mu \mathrm{m}$ deep boron diffusion is carried out to define the mass areas as well as the anchor regions (refer to figure 3(a)). A $8000 \AA$ LPCVD oxide is deposited and patterned to form the suspension beams, attaching the mass to the anchors. The oxide layer is purposefully made thicker than the design target of $7500 \AA$ since $\sim 500 \AA$ is lost in the final EDP release etch. This layer is further used as (1) bushings on the mass to decrease stiction; and (2) patterned in the gold contact regions to reduce the air-gap distance that is traversed by the contacts (refer to figure $3(b)$ ). This step is followed by evaporating and patterning a thick layer of chromium/gold (300/4000 A) to form the contacts on the mass (refer to figure $3(c)$ ). The remaining two steps are carried out on the glass substrate (refer to figure $3(d)$ ). The first step is to recess etch the glass surface by $7.5 \mu \mathrm{m}$ except in the anchoring and contact regions. This reduces the squeeze-film damping between the inertial mass and the glass substrate and reduces stiction between closely spaced structures. The second step is to pattern the photoresist, etch a $500 \AA$ recess in the glass, evaporate chromium/gold (200/1000 $\AA$ ) contact regions on the glass substrate and perform a lift-off process to pattern the metal.
The processed silicon and glass wafers are electrostatically bonded together at $370{ }^{\circ} \mathrm{C}$ at $900 \mathrm{~V}$ (figure 3(e)), and EDP etched, rinsed in de-ionized water and dried in nitrogen to release the structure (figure $3(f)$ ). The final gap separating the contacts of the threshold accelerometers is $0.25 \mu \mathrm{m}$.

\section{Interface circuit design}

The threshold accelerometer array requires an interface circuit capable of sensing and capturing the sensor switching without affecting its operation or lifetime adversely. Furthermore, the interface chip is desired to use micro-power, so that it can be used for continuous monitoring of environmental shocks or vibrations in power limited systems [1]. An ultra-low power integrated interface satisfying these requirements is designed and its general block diagram is shown in figure 4. The chip can include up to 256 channels, and four single and three triple redundant sense channels are included in the current implementation. Activation of an element of the sensor array causes an instantaneous high-to-low transition at the input of its corresponding interface channel, which is then amplified and latched. Higher reliability and fault tolerance is achieved by using channels with triple redundancy. Each of these redundant channels consists of three individual sense channels and a voter. The outputs of the redundant channels are determined by the majority function of their inputs.

The sense channels can be read individually or as an output that is a logical combination of individual channels (figure 4). This output is activated when any of a pre-programmed subset of channels sense their inputs switching. Hence, the system output can be used as an 'interrupt' or 'wake-up call' in smart low-power microsystems to trigger system re-activation from a sleep mode, or a specific service request, in case of 'an 


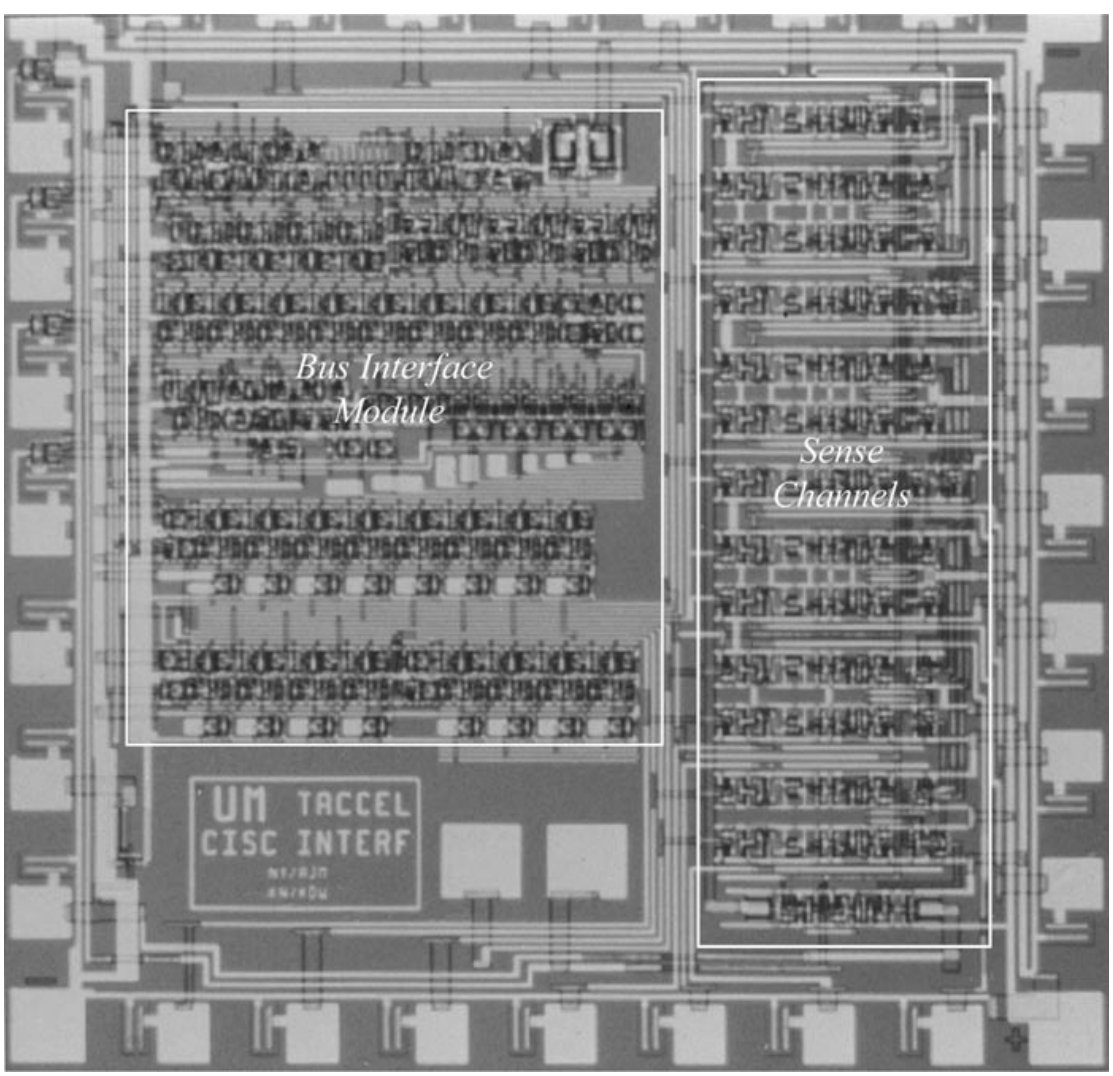

Figure 8. Photograph of the interface chip $(2.2 \mathrm{~mm} \times 2.2 \mathrm{~mm})$ fabricated with a $3 \mu \mathrm{m}$, single polysilicon, single metal, p-well CMOS process.

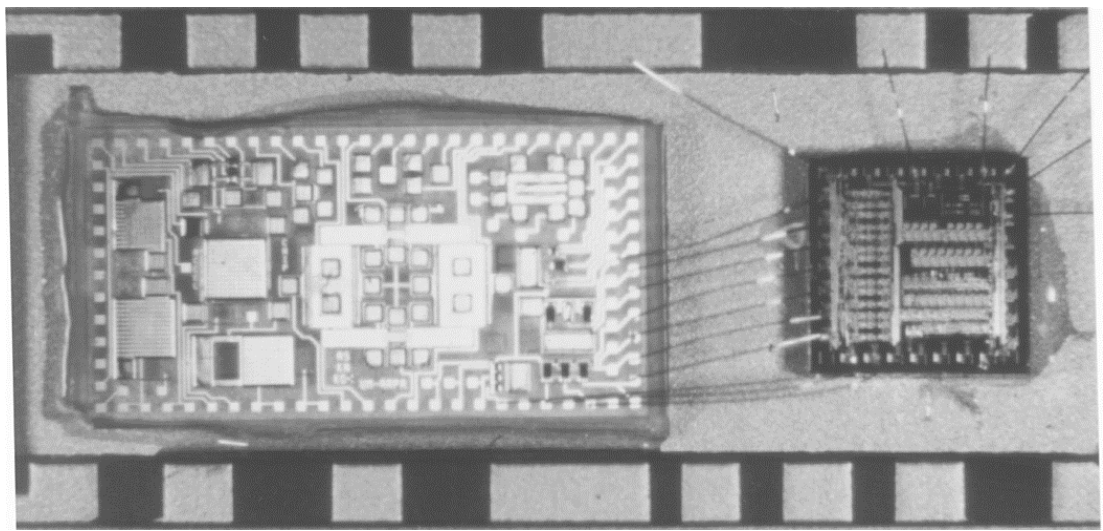

Figure 9. Photograph of the multi-chip module, with the sensor chip shown bonded to the interface chip. The threshold accelerometers are shown enclosed in the black outline.

acceleration event detection'. The ability to select a subset of the channels in this mode of operation enables the system to be user definable to specific thresholds at any instant, as required by the application. In addition, the interface chip incorporates a bus interface unit which enables it to communicate over a standard sensor bus with a microcontroller [1].

The chip includes seven channels, three of which have triple redundant sense channels. The schematic diagram of a sense channel is shown in figure 5. In the normal mode of operation, the channel is selected and enabled, i.e. transistor M3 is on and M4 is off. M1 and M2 act as a pull-up chain for the channel input. To limit the current flow through the input switch, the pull-up is designed to be weak. In fact, M1 is normally shorted, and can be added to the pull-up chain by laser trimming if a weaker pull-up is needed. This is particularly important since instantaneous large current flow through the sensor may cause its contacts to fuse together. Moreover, a limited current supply at the input reduces the overall chip power dissipation. The input high-to-low transition is amplified by a CMOS inverter (M5-M6) and latched at the channel output. The signal remains latched until a readout acknowledge signal — such as an interrupt acknowledge signal from an external microcontroller (figure 4)-is received.

The sensed signal is combined with external control signals (e.g. interrupt acknowledge), and applied as feedback to turn M3 off and M4 on. Shutting off the input channels 
provides equi-potential contacts for the sensor permitting the release of the mechanical structure. Shutting the channel current further reduces the power consumption and the likelihood of the contacts fusing together, thereby prolonging the mechanical life of the switch.

The ultra-low power operation of the interface is attained by designing for virtually no static current flow in the circuitry, and using an asynchronous design approach. Most of the circuit blocks are digital CMOS, which do not draw static current. Furthermore, application of feedback avoids steady current flow at the channel front-end. The power dissipation of the circuit designed to operate from a $5 \mathrm{~V}$ power supply was less than $10 \mu \mathrm{W}$ from transient current flow during switching events. Asynchronous design requires special attention to the various circuit delays to ensure proper operation. False input switching can be picked up due to (1) faster switching of M3 with respect to M4, and (2) low glitches at the input. The former is alleviated by designing the control logic so that the gate voltage of M3 observes an extra inverter delay. The input capacitor, $\mathrm{C}_{p}$, discharges by the switching of the input sensor, and charges up as soon as the sense channel becomes active again. Low glitches on the input occur due to timely charge up of $\mathrm{C}_{p}$ through the weak input pull-up chain, and can be sensed as fast input switching. The circuit bandwidth can be limited so that it does not respond to the fast input glitches. This has been achieved by adding a filtering capacitance, $\mathrm{C}_{f}$, to the signal path. Note that a more efficient filtering could be achieved with a smaller capacitance if it was added at the input of the channel amplifier. However, such a configuration would also increase the instantaneous current flow through the mechanical switch contacts, which is undesirable. If large bandwidth vibration signals are to be measured, the input pull-up can be designed to be stronger and the input parasitic capacitance can be minimized further. The interface chip can detect a switching transition in less than $250 \mathrm{nS}$, and hence the bandwidth of the acceleration sensing system is limited by the resonance frequency of the mechanical sense elements.

\section{Results}

The threshold accelerometers described here have been fabricated and figure 6 shows a SEM micrograph of a single device with a $50 \mu \mathrm{m} \times 50 \mu \mathrm{m} \times 15 \mu \mathrm{m}$ sized mass, two $50 \mu \mathrm{m} \times 12.5 \mu \mathrm{m} \times 0.75 \mu \mathrm{m}$ beams, and a $10 \mathrm{~g}$ threshold. A triple array with a threshold of 1.5 is illustrated in figure 7 . The yield of the threshold accelerometers, from the initial runs that were performed, was surprisingly high-well over $80 \%$, with the oxide beams proving to be very robust to wafer processing and handling. The interface chip was fabricated in-house and is shown in figure 8 . The sensor and the circuit chip were wire bonded together and packaged with the sensor chip as an MCM (multi-chip module) and is shown in figure 9.

Several tests were carried out on the threshold accelerometers to observe their switching behaviour. Probe tips were used to force the mass down to ensure switch contacting. Second, the mass elements were pulled upwards with probe tips and allowed to spring back towards the glass substrate, shorting the contacts. This was to test the latching of the transient switching of the contact mechanism. The switching of the sensors was successfully observed in each case.

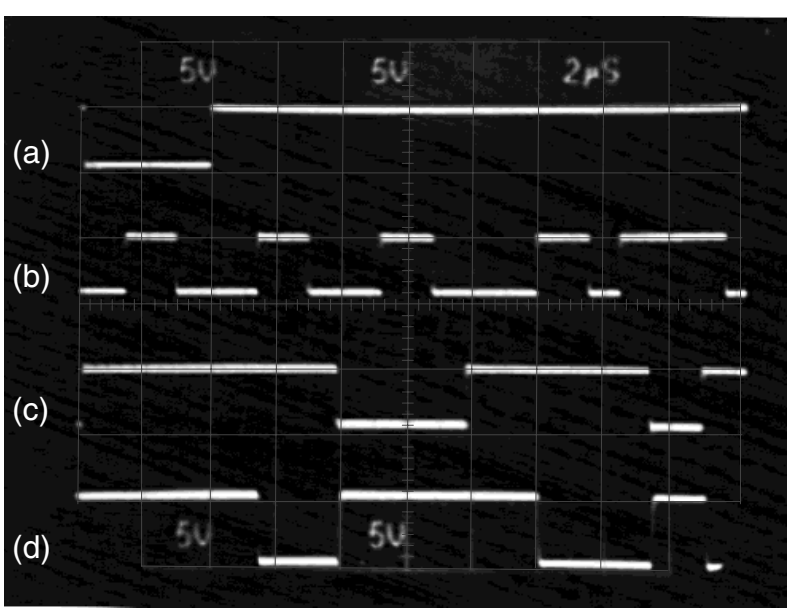

Figure 10. Test waveforms of a single channel (from top to bottom): (a) channel select, $(b)$ input switch control, $(c)$ output acknowledge and $(d)$ combined output of the channels (interrupt request signal).

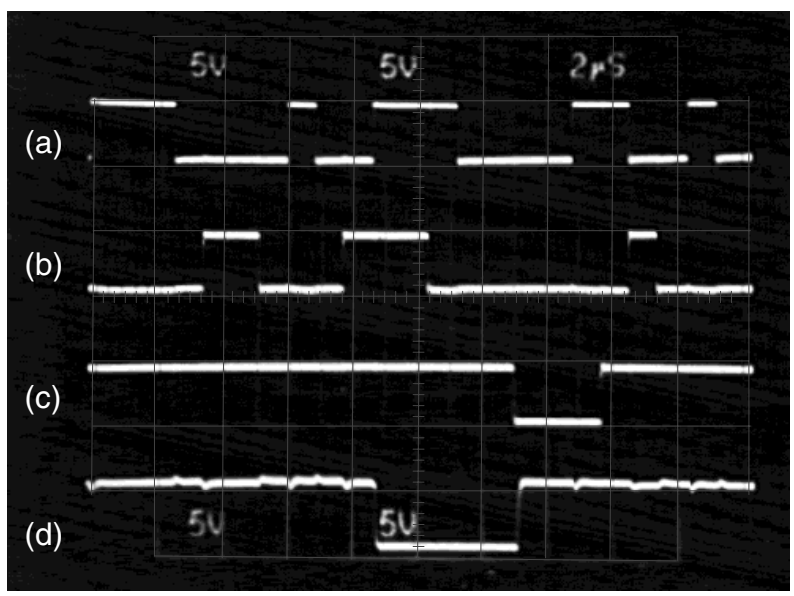

Figure 11. Test waveforms of a triple redundant channel (from top to bottom): (a) input switch control 1, $(b)$ input switch control 2, (input switch control 3-inactive), (c) output acknowledge and (d) combined output of the channels.

The chip has been fabricated using in-house facilities employing a standard $3 \mu \mathrm{m}$, single metal, double polysilicon p-well process. The chip measures $2.2 \mathrm{~mm} \times 2.2 \mathrm{~mm}$ and dissipates less than $300 \mu \mathrm{W}$ from a $5 \mathrm{~V}$ supply. Most of the supply current is due to leakage at n-epi/p-substrate and other on-chip junctions, which can be significantly reduced using a more controlled fabrication process. Using analogue switches instead of actual mechanical switches, the chip has been operated at frequencies up to $4 \mathrm{MHz}$. Figures 10 and 11 show test waveforms of single and triple redundant channels. The readout channels are enabled by a high level on their corresponding channel select input. The input analogue switches are activated by a high control signal. As is evident from figure 10, the overall output of the chip becomes low when a switching transition on at least one of the selected input channels occurs. The output is latched low until an external acknowledge signal is received and the sensing system is reset for a new set of measurements. The waveforms in figure 11 show the function of a channel with triple redundancy. In this case the overall output is inactive unless at least two of 
three input switches of the channel are triggered. Finally, the packaged sensor array and interface chip were tested together by applying a shock level to the module (tapping on the package). While switching due to the test shock level was observed, the amplitude and impact time of the applied shock were not controllable in this experiment, and hence a quantitative characterization could not be obtained.

\section{Conclusion}

We have reported the design, fabrication and testing of a threshold-acceleration-sensing microsystem using a multichip module. The threshold accelerometers were fabricated using a modified dissolved-wafer process using a silicon and glass wafer, with each sensor having a cantilevered $\mathrm{p}^{++}$inertial mass, LPCVD oxide suspension beams and gold-gold switch contacts. The sensor operation has been tested successfully for functionality.

The interface chip performed as designed. The sense channels were capable of performing up to an input switching frequency of $4 \mathrm{MHz}$. The increase in power dissipation due to leakage can be significantly lowered using a tighter fabrication process. Moreover, the packaged sensing module, consisting of the sensor array and the interface chip, has been tested for functionality.

\section{Acknowledgments}

The authors would like to thank $\mathrm{C} \mathrm{W}$ Yeh for the circuit fabrication. The bus interface design by A Mason is also appreciated. This work was supported by the Advanced Research Project Agency under contract \#J-FBI-92-149.

\section{References}

[1] Mason A, Yazdi N, Chavan A V, Najafi K and Wise K D 1998 A generic microsystem for portable wireless applications Proc. IEEE 86 1733-46

[2] Petersen K 1978 Dynamic micromechanics on silicon: techniques and devices IEEE Trans. Electron Devices $\mathbf{2 5}$ 1241-50

[3] Frobenius W D, Zeitman S A, White M H, O'Sullivan D D and Hamel R G 1972 Microminiature ganged threshold accelerometers compatible with integrated circuit technology IEEE Trans. Electron Devices 19 37-40

[4] Robinson C, Overman D, Warner R and Blomquist T 1987 Problems encountered in the development of a microscale G-switch using three design approaches Proc. 4th Int. Solid-State Sensor and Actuator Conf. (Transducers'87) Tokyo pp 410-13

[5] Go J S, Cho Y and Kwak B 1995 Acceleration microswitches with adjustable snapping threshold Proc. 8th Int. Solid-State Sensor and Actuator Conf. (Transducers'95), Stockholm pp 691-4

[6] Selvakumar A, Yazdi N and Najafi K 1996 Low power, wide range threshold acceleration sensing system Proc. IEEE Micro Electro Mechanical Systems Workshop (San Diego, CA, February) pp 186-91

[7] Gianchandhani Y and Najafi K 1991 A bulk dissolved wafer process for microelectromechanical systems Tech. Digest Int. Electron Devices Meeting (Washington, DC, 1991) pp 757-60

[8] Roylance L and Angell J 1979 A batch fabricated silicon accelerometer IEEE Trans. Electron Devices 26 1911-17

[9] Starr J B 1990 Squeeze film damping in solid-state accelerometers Dig. IEEE Solid State Sensor and Actuator Workshop (Hilton Head '90) pp 44-7

[10] Ma K, Yazdi N and Najafi K 1994 A bulk-silicon capacitive microaccelerometer with built-in overrange and force feedback electrodes Tech. Digest, Solid State Sensor and Actuator Workshop (Hilton Head 94) pp 160-3 